EPJ Web of Conferences 108,02031 (2016)

DOI: $10.1051 /$ epjconf/201610802031

(C) Owned by the authors, published by EDP Sciences, 2016

\title{
Spontaneous Coherence Effects in Quantum Dots and Quantum Wells Placed in Microcavities
}

\author{
N.E. Kaputkina ${ }^{1, a}$, Yu.E. Lozovik ${ }^{2, b}$, and M.V. Altaisky ${ }^{3, c}$ \\ ${ }^{1}$ National University of Science and Technology "MISIS", Leninsky prospect 4, Moscow, 119049, Russia \\ ${ }^{2}$ Institute of Spectroscopy RAS, Troitsk, Moscow, 142190, Russia \\ ${ }^{3}$ Space Research Institute RAS, Profsoyuznaya 84/32, Moscow, 117997, Russia
}

\begin{abstract}
The Bose-Einstein condensation temperature $T_{c}$ for a system of coupled quantum dots in a microcavity was estimated in function of the confining potential steepness, the external magnetic field strength, and the barrier layer width for indirect excitons. The effect of the magnetic field on $T_{c}$ was found to be nonmonotonic over a certain range of the control parameters. The reason is the presence of two competing mechanisms accompanying the increase of the magnetic field: (a) increase of the magnetoexciton effective mass and (b) increase of the effective confining potential steepness for quantum dots.
\end{abstract}

\section{Introduction}

The spontaneous coherence effects attract significant attention in different fields of the condensed matter physics. Lasers are the most well known manifestation of the spontaneous coherence. BoseEinstein condensation (BEC) is another manifestation of the collective coherence such that, below the critical temperature $T_{c}$, the (quasi) particles obeying Bose statistics spontaneously occupy the ground state. This happens to photons [1], to cold atoms [2], and to exciton polaritons in microcavities [3, 4]. The interaction of the excitons with photons, including polariton formation, is of particular interest, for the polaritons dramatically change the radiative properties of the quantum dots (QDs) and quantum wells (QWs) in microcavities [5-7]. In the present paper we consider the polariton dispersion law in the presence of an external magnetic field. The polariton dispersion law is essentially different from the exciton dispersion law [8]. We show that the tuning of the exciton properties by varying external magnetic field enables the control of the formation of the polaritons and of their properties.

\section{Materials and Methods}

Let us consider the conditions for polariton formation. Because of the extremely low polariton effective mass $\left(\sim 10^{-4} m_{e}[6]\right)$, the critical temperature of the Berezinskii-Kosterlitz-Thouless transition (see, e.g., [9]), (for QW exciton polaritons in a microcavity) and of the Bose-Einstein condensation

\footnotetext{
a e-mail: nataly@misis.ru

be-mail: lozovik@isan.troitsk.ru

e-mail: altaisky@mx.iki.rssi.ru
}

This is an Open Access article distributed under the terms of the Creative Commons Attribution License 4.0, which permits unrestricted use, distribution, and reproduction in any medium, provided the original work is properly cited. Article available at nttp://www.ep|-conterences.org or nttp://dx.dol.org/10.1051/ep|cont/20161080203 
(for the exciton polaritons in QDs) may be as high as $\sim 10^{2} \mathrm{~K}$. The strong polariton resonance originates in the proximity of the exciton energy and the photon energy in a microresonator formed by two parallel Bragg mirrors, see, e.g., [10, 11]:

$$
E_{\text {exc }}(k=0)+\sqrt{\left(\frac{\hbar^{2} k^{2}}{2 m^{*}}\right)^{2}+\frac{4 \pi N_{0} \hbar^{2} a}{m^{*}}\left(\frac{\hbar^{2} k^{2}}{2 m^{*}}\right)}=E_{\text {microcavity }}(k=0)+\frac{\hbar^{2} k^{2}}{2 M},
$$

where $N_{0}$ is the two-dimensional density of the exciton system, $M=\frac{\hbar \pi n l}{c L}$ is the photon "mass" in the microcavity of size $L, m^{*}$ is the effective exciton mass, $a$ is the effective radius of the magnetoexciton, $l$ is the cavity mode number, and $n$ is the refraction index. The low density gas approximation for the exciton system implies the excitons are treated as a weakly interacting Bose gas [12].

The effective mass of the spatially indirect magnetoexciton $m^{*}$ is determined by two main parameters: the strength of the external magnetic field and the width of the barrier layer [13]. The effective mass grows with the external magnetic field. Its value can be estimated by different approaches. For a sufficiently strong magnetic field, the effective mass of the magnetoexciton in its ground state can be estimated as [14]:

$$
\begin{aligned}
m^{*} & =\frac{m_{e}^{*} m_{h}^{*}}{m_{e}^{*}+m_{h}^{*}} \exp \left(-\frac{1}{2} \omega_{c} d^{2}\right)\left[\int_{d^{2}}^{\infty} \exp \left(-\frac{1}{2} t \omega_{c}\right) \frac{t^{2}-3\left(t+\frac{d^{2}}{4}\right)}{t^{5 / 2}} d t\right]^{-1} \\
& =\frac{m_{e}^{*} m_{h}^{*}}{m_{e}^{*}+m_{h}^{*}} \frac{4 d \sqrt{\omega_{c}}}{\sqrt{2 \pi} \exp \left(\frac{d^{2} \omega_{c}}{2}\right)\left[\left(d^{3} \omega_{c}^{2}-12 d \omega_{c}-4 d\right)\left(\operatorname{erf}\left(d \sqrt{\frac{\omega_{c}}{2}}\right)-1\right)\right]-26 \sqrt{\omega_{c}}+2 d^{2} \omega_{c}^{\frac{3}{2}}},
\end{aligned}
$$

where $m_{e(h)}^{*}$ is the electron (hole) effective mass, $\omega_{c}$ and $d$ are the cyclotronic frequency and the barrier width in dimensionless units

$$
E_{0}=\frac{2 m_{e}^{*} e^{4}}{\hbar^{2} \varepsilon^{2}}, \quad a_{0}=\frac{\hbar^{2} \varepsilon}{2 m^{*} e^{2}}, \quad \alpha_{0}=\frac{E_{0}}{a_{0}^{2}}, \quad \omega_{c_{0}}=\frac{2 m^{*} e^{4}}{\varepsilon^{2} \hbar^{3}},
$$

for the energy, length, confining potential steepness, and cyclotronic frequencies, respectively. The choice of these variables is related to the energy and size of the two-dimensional exciton [15].

For the excited energy levels the effective mass of the magnetoexciton may be negative for small $k$ [14]. This may result in a nonmonotonic dispersion law for the excited states of the exciton polaritons $[13,16]$.

We also consider the indirect two-dimensional excitons in two vertically coupled two-dimensional quantum dots ("natural", or artificial) with parabolic confinement potentials for the electrons and the holes, respectively: $U_{e, h}=\alpha_{e, h} r_{e, h}^{2}$. In particular, the localization of the excitons may be realized due to the confining potentials, $U_{e}$ or $U_{h}$. The relative motion energy levels of the electron and the hole [17]

$$
\left(\Delta_{r}+E_{\mathrm{rel}}-\alpha_{\mathrm{eff}} r^{2}+\gamma \frac{m \omega_{c}}{2}+\frac{1}{\left(r^{2}+d^{2}\right)^{1 / 2}}\right) \psi(r)=0, \quad r=r_{e}-r_{h},
$$

with $\gamma=\frac{m_{h}^{*}-m_{e}^{*}}{m_{h}^{*}+m_{e}^{*}}$ and $m \in \mathbb{Z}$ being the magnetic quantum number, increase monotonically with the growth of the effective steepness parameter

$$
\alpha_{\mathrm{eff}}=\frac{m_{e}^{*} m_{h}^{*}\left(\omega_{c} / 4\right)^{2}+m_{h}^{* 2} \alpha_{e}+m_{e}^{* 2} \alpha_{h}}{\left(m_{e}^{*}+m_{h}^{*}\right)^{2}} .
$$




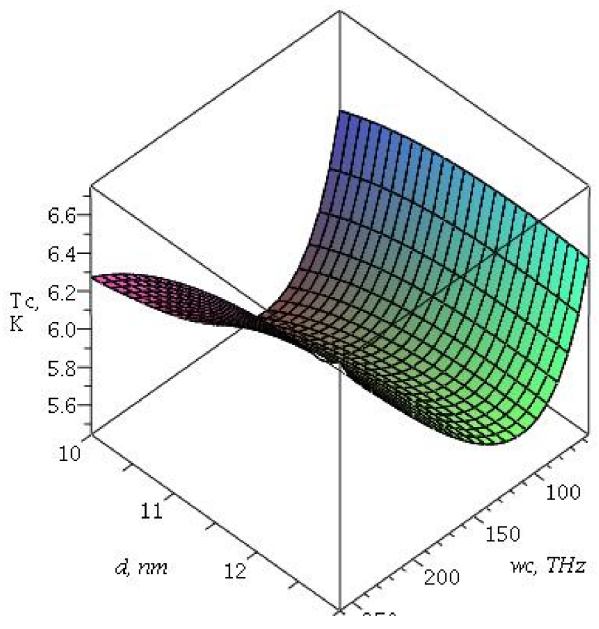

a)

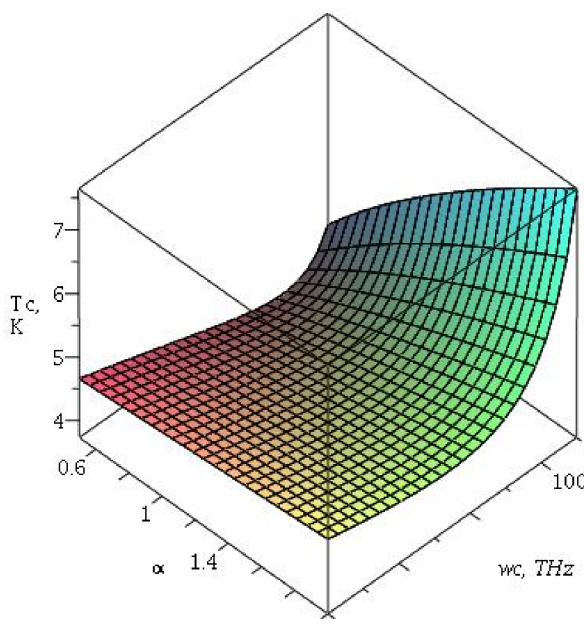

b)

Figure 1. Dependence of the critical temperature of the exciton polariton Bose-Einstein condensation: a) on the magnetic field (cyclotronic frequency $\omega_{c}, \mathrm{THz}$ ) and on the barrier width $d, \mathrm{~nm}$, at the fixed value of $\alpha_{\text {eff }}=1.0$; b) on the magnetic field (cyclotronic frequency $\omega_{c}, \mathrm{THz}$ ) and on the confining parameter $\alpha_{\text {eff }}$ at the fixed barier width $d=8 \mathrm{~nm}$

If $\alpha_{e, h}$ is large enough (due to either a strong confining potential, or a large inter-layer distance), the e-h interaction is small in comparison to the other parameters, and the relative motion energies are linear in $\sqrt{\alpha_{\text {eff }}}$. For a super-strong magnetic field the energy levels asymptotically approach the Landau energy levels defined in the case without any parabolic confinement.

For the ideal two-dimensional Bose gas the Bose-Einstein condensation is forbidden, but in a two-dimensional trap in a parabolic confining potential the Bose-Einstein condensation may occur at $T<T_{c}$, where

$$
T_{c}=\left(\frac{3 \alpha_{\mathrm{eff}} N_{0} s}{2 M_{\mathrm{eff}}}\right)^{\frac{1}{2}} \frac{\hbar}{k_{B} \pi},
$$

with $s$ denoting the spin degeneration, $s=2$ for bright excitons [18]. The value of the polariton effective mass $M_{\text {eff }}$ near the center of the trap is close to the exciton effective mass (2) [19]. For the fairly small steepness of the confining potential the critical parameters of the Bose-Einstein condensation can be estimated in the local density approximation [19]. The polariton effective mass can be estimated using the Bogoliubov approximation [20].

\section{Results and Conclusions}

In the present work we studied the quantum dot and the quantum well systems embedded in microcavities. Exciton polariton spontaneous coherence effects were considered at low temperatures. In contrast to the confinementless unbounded two-dimensional systems, the Bose-Einstein condensation of the exciton polaritons takes place for coupled quantum dots in microcavity.

The dependence of the critical temperature $T_{c}$ (3) on the barrier layer width $(d)$ and on the strength of magnetic field $\left(\omega_{c}\right)$ is shown Fig. 1. It presents the results of calculations performed for the GaAs 
based quantum dots with the effective electron mass $m_{e}^{*}=0.067 m_{e}$ and the dielectric constant value $\epsilon=10$. The two-dimensional density of the excitons near the center of the trap $N_{0}=1.2 \times 10^{9} \mathrm{~cm}^{-2}$ was taken from [19].

The higher the confinement steepness, the higher is the critical temperature. The increase of the barrier width also favours the increase of the critical temperature. The effect of the magnetic field on the value of the critical temperature may be nonmonotonic in a certain range of control parameters because of the presence of two competing mechanisms: the increase of the magnetoexciton effective mass and the increase of the effective confining potential steepness.

Since the exciton polariton spectrum, a linear spectrum, meets the Landau superfluidity criterium, the superfluidity of the polaritons may occur in a microcavity.

\section{Acknowledgements}

The research was supported in part by RFBR projects 13-07-00409, 14-02-00739 and by the Ministry of Education and Science of the Russian Federation in the framework of Increase Competitiveness Program of "MISIS".

\section{References}

[1] J. Klaers, J. Schmitt, F. Vewinger, M. Weitz, Nature 468, 545 (2010)

[2] M.H. Anderson, J.R. Ensher, M.R. Matthews, C.E. Wieman, E.A. Cornell, Science 269, 198 (1995)

[3] H. Deng, G. Weihs, C. Santori, J. Bloch, Y. Yamamoto, Science 298, 199 (2002)

[4] H. Deng, H. Haug, Y. Yamamoto, Rev. Mod. Phys. 82, 1489 (2010)

[5] S. Pau, H. Cao, J. Jacobson, G. Björk, Y. Yamamoto, A. Imamoğlu, Phys. Rev. A 54, R1789 (1996)

[6] R. Balili, V. Hartwell, D. Snoke, L. Pfeiffer, K. West, Science 316, 1007 (2007)

[7] J. Kasprzak, a.o., Nature 443, 409 (2006)

[8] S. Jorda, Phys. Rev. B 51, 10185 (1995)

[9] J. Jose, 40 Years of Berezinskii-Kosterlitz-Thouless Theory (World Scientific (Singapore), 2013)

[10] N.E. Kaputkina, Y.E. Lozovik, Physica status solidi (c) 3, 3500 (2006)

[11] V. Timoffeev, Semiconductors 46, 843 (2012)

[12] N. Bogolyubov, J.Phys.(USSR) 11, 23 (1947)

[13] N. Kaputkina, Y. Lozovik, Phys.Stat.Sol.(c) 6, 20 (2009)

[14] N. Kaputkina, Y. Lozovik, Physica E 26, 291 (2005)

[15] N. Kaputkina, Y. Lozovik, Physica E 12, 323 (2002)

[16] N. Kaputkina, Y.E. Lozovik, Phys. Stat. Sol. (c) 3, 3500 (2006)

[17] Y.E. Lozovik, N.E. Kaputkina, Physica status solidi (b) 207, 147 (1998)

[18] V. Bagnato, D. Kleppner, Phys. Rev. A 44, 7439 (1991)

[19] O. Berman, Y. Lozovik, D. Snoke, Phys. Rev. B 77, 155317 (2008)

[20] Y. Lozovik, A. Semenov, Theoretical and Mathematical Physics 154, 319 (2008) 